07

\title{
Влияние резонансного взаимодействия поверхностных магнитостатических волн с обменными модами на генерацию ЭДС в структурах YIG/Pt
}

\author{
(C) М.Е. Селезнев, ${ }^{1,2}$ Ю.В. Никулин, ${ }^{1,2}$ В.К. Сахаров, ${ }^{1}$ Ю.В. Хивинцев, ${ }^{1,2}$ А.В. Кожевников, ${ }^{1}$ С.Л. Высоцкий, ${ }^{1,2}$ \\ Ю.А. Филимонов ${ }^{1,2}$
}

${ }^{1}$ СФИРЭ им. В.А. Котельникова РАН,

410019 Саратов, Россия

${ }^{2}$ Саратовский государственный университет им. Н.Г. Чернышевского,

410012 Саратов, Россия

e-mail: mixanich94@mail.ru

Поступило в Редакцию 30 апреля 2021 г.

В окончательной редакции 30 апреля 2021 г.

В окончательной редакции 30 апреля 2021 г.

Исследованы особенности генерации ЭДС $(U)$ в тонкопленочных структурах Pt/YIG, обусловленные эффектами гибридизации бегущих поверхностных магнитостатических волн с обменными объемными модами пленки YIG. Показано, что на частотах дипольно-обменных резонансов величина ЭДС увеличивается по сравнению с соседними частотными областями. Проведена оценка вольт-ваттной чувствительности для структур Pt/YIG с различными геометриями Pt-микрополосок.

Ключевые слова: спинтроника, спиновые волны, генерация ЭДС, эффект увлечения электронов, дипольнообменные резонансы.

DOI: $10.21883 / J T F .2021 .10 .51363 .136-21$

\section{Введение}

Исследование распространения спиновых волн и генерации ЭДС в структурах магнитный диэлектрик-металл представляет интерес для создания энергоэффективной элементной базы на принципах магноники и спинтроники [1-3]. Одной из задач данных направлений является изучение механизмов и улучшение эффективности преобразования спиновых волн в электрический ток на границе феррит-проводник и в объеме проводника. В этой связи наиболее широко исследуются структуры на основе пленки железоиттриевого граната (YIG) и платины $(\mathrm{Pt})$ - металла с сильной спин-орбитальной связью. В такого рода структурах электроны проводимости металла чувствительны к намагниченности пленки YIG за счет обменного и спин-орбитального взаимодействий, что приводит к генерации ЭДС при распространении спиновой волны за счет обратного спинового эффекта Холла [4] или эффекта увлечения электронов волной в объеме проводника [5-7]. При этом исследование влияния дипольно-обменных резонансов на генерируемую ЭДС при распространении ПМСВ в структурах Pt/YIG ранее не проводилось.

В настоящей работе исследовались особенности генерации ЭДС $(U)$ в тонкопленочных структурах Pt/YIG для случая, когда в пленке YIG бегущие поверхностные магнитостатические волны (ПМСВ) гибридизуются с обменными объемными модами пленки YIG.

\section{1. Исследованные структуры и методика эксперимента}

Для изготовления структур использовалась пленка YIG толщиной $d \sim 900 \mathrm{~nm}$ с эффективной намагниченностью насыщения $4 \pi M^{\text {eff }} \sim 1800 \mathrm{G}$ и шириной линии ферромагнитного резонанса $2 \Delta H \sim 0.6 \mathrm{Oe}$, выращенная на подложке гадолиний-галлиевого граната (GGG) (пленка YIG отмечена цифрой 5 рис. 1). На поверхности пленки YIG методом магнетронного распыления была выращена пленка $\mathrm{Pt}$ толщиной $t \sim 9 \mathrm{~nm}$, из которой методами фотолитографии и ионного травления формировались структуры трех типов (1-3, рис. 1). Структура 1 (рис. $1, a)$ имела на поверхности YIG одиночную микрополоску $\mathrm{Pt}$ длиной $L \sim 220 \mu \mathrm{m}$ и шириной $W \sim 200 \mu \mathrm{m}$ и удельным сопротивлением $\rho \sim 0.41 \cdot 10^{-6} \Omega \cdot \mathrm{m}\left(R_{\square} \sim 0.41 \cdot 10^{2} \Omega / \mu \mathrm{m}\right)$. В структуре 2 (рис.1, b) на поверхности пленки YIG формировалась одиночная микрополоска $\mathrm{Pt}$ шириной $W \sim 40 \mu \mathrm{m}, L \sim 220 \mu \mathrm{m}$ и $\rho \sim 0.324 \cdot 10^{-6} \Omega \cdot \mathrm{m}$ $\left(R_{\square} \sim 0.41 \cdot 10^{2} \Omega / \mu \mathrm{m}\right)$. Структура 3 (рис. $\left.1, c\right)$ содержала три последовательно соединенных медным проводником микрополоски Pt с $L \sim 220 \mu \mathrm{m}, W \sim 40 \mu \mathrm{m}$ и $\rho \sim 0.32 \cdot 10^{-6} \Omega \cdot / \mu \mathrm{m}\left(R_{\square} \sim 0.32 \cdot 10^{2} \Omega / \mu \mathrm{m}\right)$. Микрополоски Рt формировались между интегрированными на поверхности пленки YIG медными микроантеннами (1 и 2), которые располагались на расстоянии $250 \mu \mathrm{m}$ друг от друга и имели апертуру (длину) $a \sim 250 \mu$ m и ширину $b \sim 4 \mu \mathrm{m}$. Для измерения генерируемой в структурах 
$a$

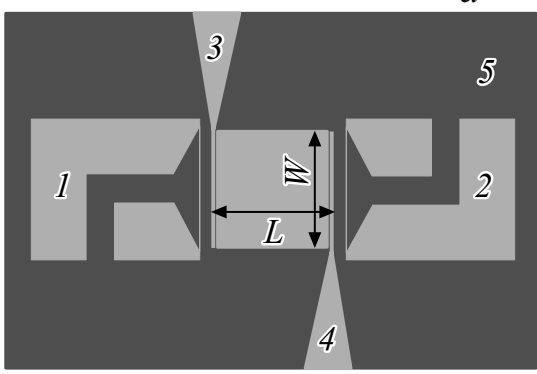

$b$

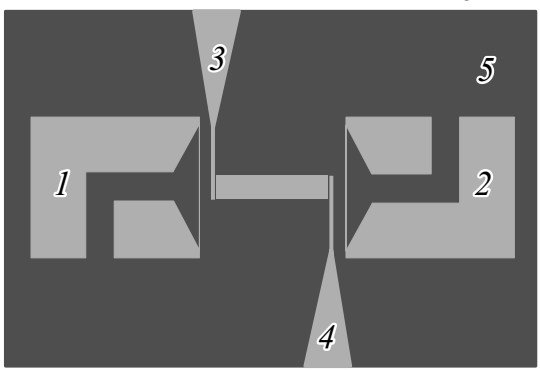

C

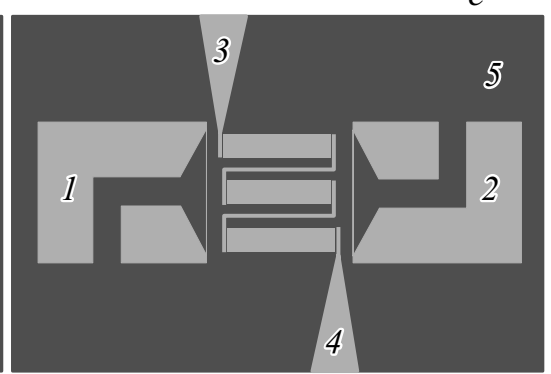

Рис. 1. Микроструктуры Pt/YIG в виде одной Рt микрополоски длиной $L \sim 220 \mu \mathrm{m}$ и шириной $W \sim 200 \mu \mathrm{m}(a)$; одной Pt микрополоски с $L \sim 220 \mu \mathrm{m}$ и $W \sim 40 \mu \mathrm{m}(b)$ и трех последовательно соединенных Рt микрополосок с $L \sim 220 \mu \mathrm{m}$ и $W \sim 40 \mu \mathrm{m}$ каждая $(c)$. 1,2 - возбуждающие микроантенны; 3, 4 - медные контакты для измерения генерируемого напряжения; 5 - поверхность пленки YIG.

ЭДС на концах Pt микрополосок по всей ширине $W$ формировались медные контакты (3 и 4).

При проведении исследований структуры размещались между полюсами электромагнита таким образом, что магнитное поле $H \sim 939$ Ое было направлено касательно поверхности пленки YIG и перпендикулярно длине $L$ микрополосок, что соответствовало геометрии возбуждения ПМСВ. Измерение частотных зависимостей модуля и фазы коэффициентов прохождения $\left(S_{12}\right)$ и отражения $\left(S_{22}\right)$ спиновых волн осуществлялось с использованием векторного анализатора цепей, подключенного к интегрированным на поверхности YIG микроантеннам 1 и 2 с помощью СВЧ микрозондов (рис. 1).

Исследование частотной зависимости ЭДС $U(f)$, генерируемой при распространении ПМСВ, производилось с использованием селективного вольтметра, который подключался к контактам 3 и 4 (рис. 1). При этом для обеспечения работы вольтметра на входную антенну подавался СВЧ сигнал с импульсной модуляцией $(11.33 \mathrm{kHz})$, что также способствовало уменьшению влияния нагрева на регистрируемую ЭДС.

\section{2. Результаты и обсуждение}

На рис. 2, $а$ показаны частотные зависимости амплитуды коэффициента прохождения $S_{12}(f)$, полученные при входной мощности $P \sim-20 \mathrm{dBm}$ для структур $1-3$ (кривые 1-3 соответственно) и пленки YIG (кривая 4). Наименьшие значения $S_{12}$ по сравнению с пленкой YIG наблюдались для структуры 3 , что можно объяснить не только поглощением ПМСВ полосками $\mathrm{Pt}$, но и влиянием медных контактов к полоскам Pt на эффективность возбуждения ПМСВ и условия распространения. Наибольшие значения $S_{12}$ регистрировались для структуры 2 , где как ширина $W$ микрополоски $\mathrm{Pt}$, так и длина медных контактных дорожек к $\mathrm{Pt}$ была наименьшей среди структур $1-3$.

На рис. 2, $b$ приведены рассчитанные аналогично [8] на основе полученных фазочастотных зависимостей $\varphi(f)$ дисперсионные характеристики $k(f)$, где $k-$ волновое число ПМСВ. В структуре 2 также наблюдались ПМСВ с наибольшими значениями $k \sim 11000 \mathrm{~cm}^{-1}$, которые были близки к измеренным значениям $k \sim 12000 \mathrm{~cm}^{-1}$ для структуры без платины (кривые 2 и 4 на рис. $2, b$ ). Следует отметить, что построенные дисперсионные характеристики $k(f)$ структур $1-3$ в области волновых чисел $k \leq 6000 \mathrm{~cm}^{-1}$ практически не отличаются от расчета для дипольных ПМСВ Деймона-Эшбаха в свободной пленке с выбранными параметрами (кривая 5 на рис. 2,b). Однако для $k>6000 \mathrm{~cm}^{-1}$ результаты измерений и расчета заметно расходятся. Такое поведение измеренной дисперсии указывает на неоднородность параметров пленки по толщине и может объясняться ростом эффективной намагниченности пленки в приповерхностном слое.

Необходимо отметить наличие частот, на которых уровень $S_{12}$ значительно (на 5-15 dB) уменьшается (см. выделенные звездочками участки характеристики $S_{12}(f)$ на кривой 4 на рис. $2, a)$. Такие особенности в спектрах прохождения ПМСВ отражают резонансное взаимодействие ПМСВ с обменными объемными модами пленочного волновода и связаны с резонансным ростом потерь ПМСВ [9]. При этом в дисперсионной зависимости $k(f)$ на частотах дипольно-обменных резонансов возникают аномальные участки дисперсии [9], как это показано на вставке к рис. $2, b$ для резонансной частоты $\sim 4.645 \mathrm{GHz}$.

На рис. 2,c представлены частотные зависимости генерируемой ЭДС $U(f)$ для исследуемых структур при входной мощности накачки $P_{\text {in }} \sim-5 \mathrm{dBm}$. Можно видеть, что зависимости $U(f)$ для всех исследованных структур демонстрируют резонансный рост сигнала $U$ на частотах, соответствующих частотам дипольнообменных резонансов. Чтобы проиллюстрировать сказанное, на рис. $3, a, b$ для узкой полосы частот приведены участки зависимости $S_{12}(f)$ и $U(f)$, содержащие резонансные особенности. Видно также, что на частотах $f>5 \mathrm{GHz}$ величина генерируемой ЭДС заметно падает.

Отмеченное снижение $U$ на частотах $f>5 \mathrm{GHz}$ можно связать с уменьшением коэффициента преобразова- 

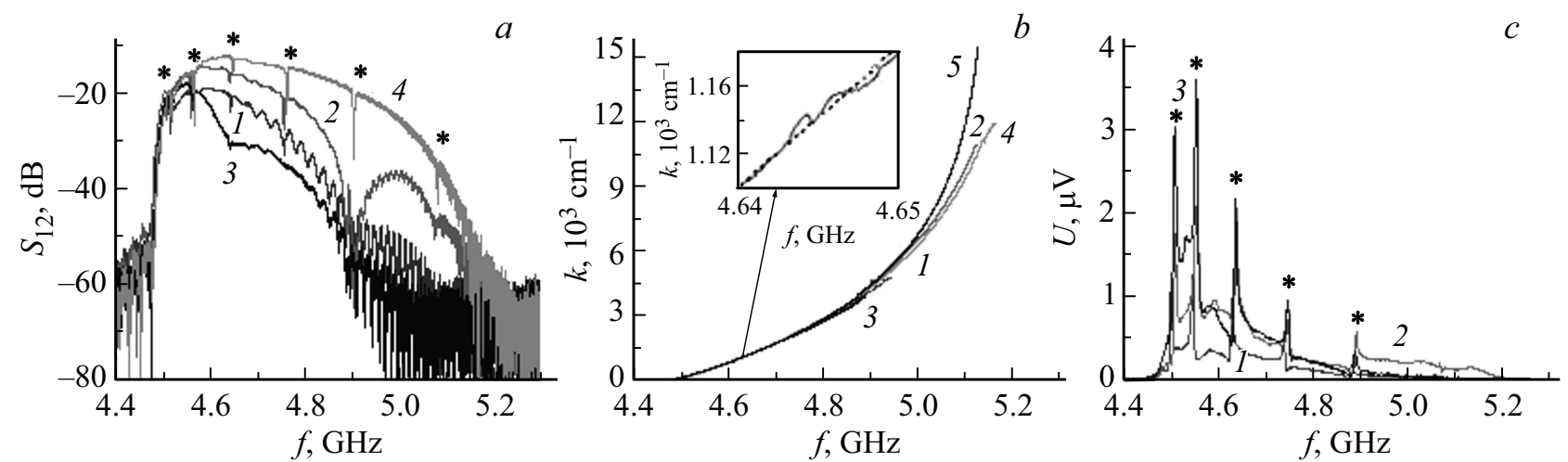

Рис. 2. Частотные зависимости коэффициента прохождения $S_{12}(a)$, волнового числа $k$ ПМСВ $(b)$ и генерируемой ЭДС $(c)$ при поле $H \sim 939$ Ое. $1-3$ - результаты для структур 1-3 соответственно; 4 - результаты для пленки YIG; 5 - теоретический расчет дисперсии ПМСВ. На вставке к рис. 2, $b$ показаны дисперсионные зависимости в окрестности частоты $\sim 4.645 \mathrm{GHz}$, измеренные для пленки YIG (сплошная линия) и рассчитанные для дипольной ПМСВ (пунктирная линия).
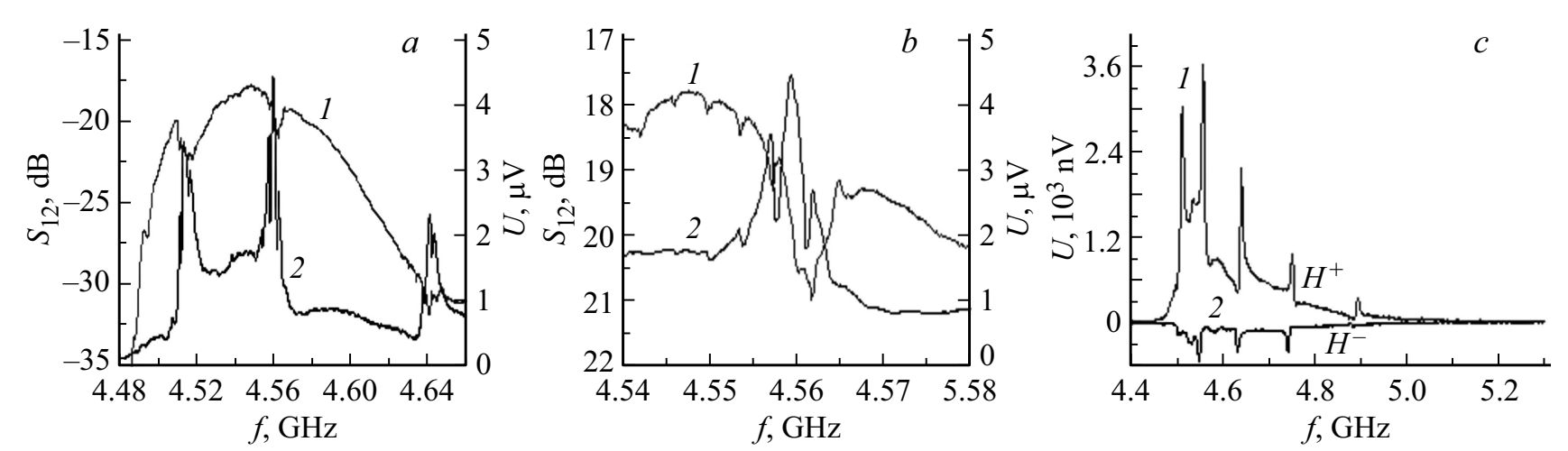

Рис. 3. Частотные зависимости коэффициента прохождения $S_{12}(1)$ и генерируемой ЭДС $U(f)(2)$ структуры 3 на частотном диапазоне вблизи нескольких дипольно-обменных резонансов $(a)$ и в окрестности одного резонанса $(b) ; c-$ частотные зависимости генерируемой ЭДС $U(f)$ при распространении в одном и том же направлении ПМСВ вдоль границ Рt/YIG $(1)$ и YIG/GGG (2), что достигалось сменой направления подмагничивания $H^{+}$на противоположное $H^{-}$. Магнитное поле $H \sim 939$ Ое.

ния $K(f)$ антеннами входной мощности $P_{i n}(f)$ в мощность ПМСВ $P(f)=K(f) \cdot P_{\text {in }}(f)$ для ПМСВ с длиной волны $\lambda<2 \cdot b\left(k>\pi / b \sim 8000 \mathrm{~cm}^{-1}\right)$. Это подтверждается расчетом зависимостей $K(f)$ для структур $1-3$ с помощью соотношения

$$
K(f)=\frac{P(f)}{P_{\text {in }}(f)}=10^{\left[S_{22}(f, h) / 10\right]}-10^{\left[S_{22}\left(f, H^{*}\right) / 10\right]},
$$

где $S_{22}(f, H)$ и $S_{22}\left(f, H^{*}\right)$ - коэффициенты отражения падающей мощности от входной антенны при полях $H \sim 939$ Ое и $H^{*} \sim 2473$ Ое, при которых антенна возбуждает и не возбуждает ПМСВ на выбранной частоте $f$ соответственно. Из рис. 3, а можно видеть, что значения $K(f)$ заметно снижаются на частотах $f>5 \mathrm{GHz}$, на которых ПМСВ характеризуются волновыми числами $k>\pi / b \sim 8000 \mathrm{~cm}^{-1}$ (рис. $\left.2, b\right)$.

Для выяснения механизма генерации ЭДС обратимся к рис. 3,c, где показаны частотные зависимости генерируемой ЭДС $U(f)$ структуры 3 при распространении ПМСВ в одном и том же направлении, но при локализации полей ПМСВ на границах Pt/YIG и YIG/GGG.
Смена поверхности, вдоль которой распространялась ПМСВ, достигалась за счет смены направления внешнего магнитного поля $H^{+}$на противоположное $H^{-}$. При этом наблюдалась смена знака генерируемой ЭДС $U(f)$, что может свидетельствовать о том, что основным механизмом генерации ЭДС в структурах Pt/YIG является обратный спин-Холл эффект [3], а не эффект увлечения или термический нагрев структуры [5-7]. Действительно, знак ЭДС, связанный с перечисленными эффектами, не должен меняться при смене направления поля [5]. Таким образом, можно сделать вывод, что увеличение сигнала ЭДС на частотах дипольнообменных резонансов может быть связано с усилением спинового тока на границе Pt/YIG обменными модами, распространяющимися по толщине пленки YIG.

Обсудим теперь вольт-ваттную чувствительность $S(f)=U(f) /\left(P_{\text {in }} \cdot K(f)\right)$ и связь генерируемого сигнала $U(f)$ от ширины и количества микрополосок платины в рассмотренных структурах (рис. 1). При механизме спиновой накачки величина протекаемого спинового тока $j_{s}$ через интерфейс Pt/YIG будет определяться погонной 

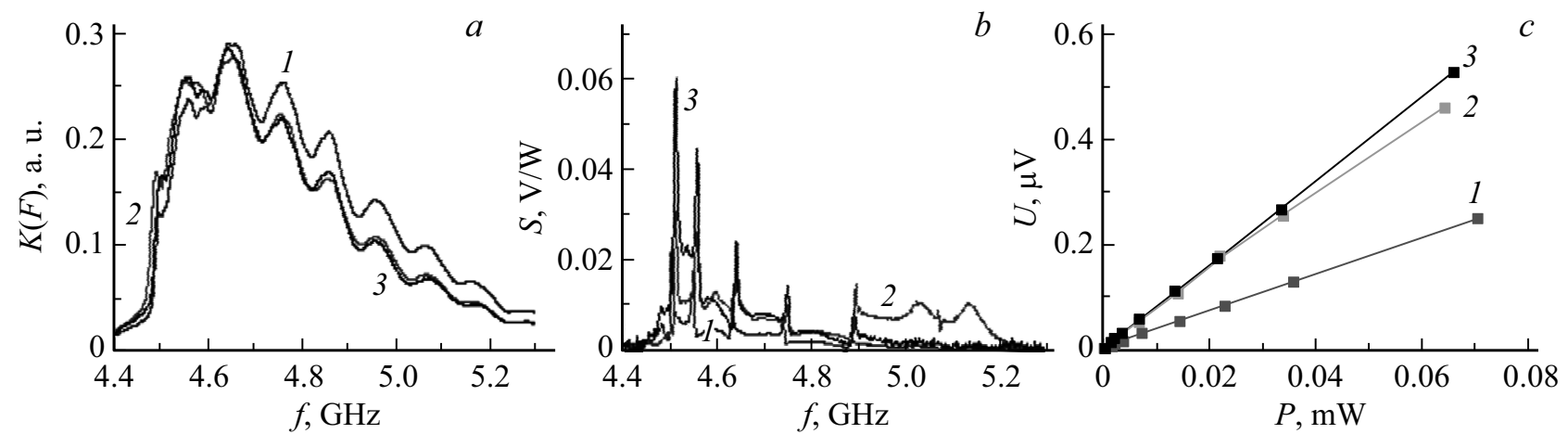

Рис. 4. Рассчитанные частотные зависимости коэффициента преобразования $K(f)(a)$ и чувствительности $S(f)(b)$; $c-$ измеренная зависимость $U(P)$ на частоте $4.7 \mathrm{GHz}$ и поле $H \sim 939$ Ое. $1-3$ - результаты для структур $1-3$ соответственно.

(по длине $a$ антенны) мощностью ПМСВ $P^{\prime}=P / a$ и площадью $S=W \cdot L$ контакта Pt/YIG: $j_{s} \sim P^{\prime} \cdot S$. Генерируемый за счет обратного спин-Холл эффекта сигнал ЭДС $U \sim j_{s}, R_{\mathrm{Pt}} \sim P^{\prime} \cdot \rho \cdot L^{2} / t$, где $R_{\mathrm{Pt}}=\rho \cdot L /(W \cdot t)-$ сопротивление пленки платины. При этом генерируемая ЭДС не зависит от ширины пленки Pt аналогично тому, как ранее наблюдалось для ЭДС увлечения в структуpax InSb/YIG [10]. Применительно к рассматриваемым структурам 1-3 это должно проявляться в одинаковых значениях $U$ для структур 1 и 2 и в три раза большей величине ЭДС для структуры 3.

На рис. 4, $b$ приведены рассчитанные частотные зависимости чувствительности структур $S(f)=$ $=U(f) /\left(P_{i n} \cdot K(f)\right)$. Можно видеть, что на частотах $f \sim 4.5-4.55 \mathrm{GHz}$ чувствительность структуры 3 достигала значений $S \sim 0.023 \mathrm{~V} / \mathrm{W}$, а на частотах дипольнообменных резонансов $S \sim 0.06 \mathrm{~V} / \mathrm{W}$, в то время как чувствительность структуры 2 на тех же частотах достигала значений $S \sim 0.011$ и $0.03 \mathrm{~V} / \mathrm{W}$ соответственно, что в среднем в два раза меньше. Чувствительность $S(f)$ структуры 1 практически на всем частотном диапазоне была меньше в 2-3 раза по сравнению с $S(f)$ структур 2 и 3. Однако на частотах $f>4.55 \mathrm{GHz}$ наблюдались схожие значения чувствительностей структур 2 из.

На рис. 4, с приведены зависимости ЭДС на фиксированной частоте возбуждения ПМСВ $f=4.7 \mathrm{GHz}$. Можно видеть, что значения ЭДС для структур 2 и 3 близки. Одной из причин совпадения зависимостей $S(f)$ для структур 2 и 3 на частотах $f>4.55 \mathrm{GHz}$ может быть влияние контактных дорожек из $\mathrm{Cu}$ на характер распространения ПМСВ в структуре 3 и распределение погонной мощности $P^{\prime}$ в плоскости структуры.

Следует отметить, что обсуждаемый здесь эффект обменных осцилляций ЭДС в структуре $\mathrm{Pt}(9 \mathrm{~nm}) / \mathrm{YIG}(900 \mathrm{~nm})$ принципиально отличается от обменных осцилляций ЭДС увлечения в структуре $\mathrm{InSb} / \mathrm{YIG}$ [7]. Действительно, в работе [7] ЭДС в структурах InSb/YIG резонансно уменьшалась на частотах дипольно-обменных резонансов и имела вид аналогичный виду зависимости $S_{12}(f)$, показанный кривой 4 на рис. $2, a$ или кривыми 1 на рис. $3, a, b$. Кроме того, согласно теории увлечения электронов $[5,6]$, бегущими спиновыми волнами сигнал ЭДС $U(f)$ должен увеличиваться с ростом $k$. Этот эффект может заметно компенсировать падение коэффициента $K(f)$ в коротковолновой части спектра ПМСВ и позволять детектировать ЭДС вблизи коротковолновой границы спектра ПМСВ [4-7].

\section{Заключение}

Исследована генерация ЭДС $U(f)$ в структурах $\operatorname{Pt}(9 \mathrm{~nm}) / \mathrm{YIG}(900 \mathrm{~nm})$ при распространении в пленке YIG ПМСВ в условиях формирования дипольнообменных резонансов. Обнаружено, что на частотах дипольно-обменных резонансов ПМСВ генерируемая ЭДС $U(f)$ может увеличиваться в несколько раз. При этом вольт-ваттная чувствительность на частотах дипольно-обменных резонансов в рассмотренном случае достигала значений $S \sim 0.06 \mathrm{~V} / \mathrm{W}$, что почти в три раза выше, чем в соседних частотных областях.

\section{Финансирование}

Работа выполнена в рамках государственного задания при частичной финансовой поддержке Российского фонда фундаментальных исследований (проекты № 19-3790099, 20-07-00968).

\section{Конфликт интересов}

Авторы заявляют, что у них нет конфликта интересов.

\section{Список литературы}

[1] A. Li, W. Zhang, V. Tyberkevych, W.K. Kwok, A. Hoffmann, V. Novosad. J. Appl. Phys., 128 (13), 130902 (2020).

[2] A. Hirohata, K. Yamada, Y. Nakatani, I.L. Prejbeanu, B. Diény, P. Pirro, B. Hillebrands. J. Magn. Magn. Mater., 509, 166711 (2020).

[3] V.E. Demidov, S. Urazhdin, A. Anane, V. Cros, S.O. Demokritov. J. Appl. Phys., 127 (17), 170901 (2020). 
[4] U.V. Nikulin, M.E. Seleznev, Y.V. Khivintsev, V.K. Sakharov, E.S. Pavlov, S.L. Vysotskii, A.V. Kozhevnikov, Y.A. Filimonov. Semiconductors, 54, 1721 (2020).

[5] Ю.В. Гуляев, П.Е. Зильберман, А.О. Раевский. ЖЭТФ, 76, (5), 1593 (1979).

[6] А.С. Бугаев, О.Л. Галкин, Ю.В. Гуляев, П.Е. Зильберман. Письма в ЖТФ, 8, 485 (1982).

[7] Г.Т. Казаков, А.Г. Сухарев, Ю.А. Филимонов, С.К. Нурджанова, Б.П. Нам, А.С. Хе. РЭ, 23 (4), 807 (1988).

[8] Y. Khivintsev, Y. Filimonov, S. Nikitov. Appl. Phys. Lett., 106, 052407 (2015)

[9] Ю.В. Гуляев, А.С. Бугаев, П.Е. Зильберман, И.А. Игнатьев, А.Г. Коновалов, А.В. Луговской, А.М. Медников, Б.П. Нам, Е.И. Николаев. Письма в ЖЭТФ, 30, 600 (1979).

[10] С.Л. Высоцкий, Г.Т. Казаков, А.Г. Сухарев, Ю.А. Филимонов. Бюлл. изобр. 1 (1988). 